\title{
ANÁLISIS DE VARIABLES PRODUCTIVAS Y SOCIOEMPRESARIALES DE PRODUCTORES DE AGUACATE HASS DE DOS MUNICIPIOS DEL CAUCA PARA LA CREACIÓN DE INDICADORES PARA LA TOMA DE DECISIONES DEL SECTOR RURAL
}

\author{
ANALYSIS OF PRODUCTIVE AND SOCIO-BUSINESS VARIABLES \\ OF AVOCADO HASS PRODUCERS IN TWO MUNICIPALITIES \\ OF CAUCA FOR THE CREATION FOR THE CREATION OF \\ DECISION-MAKING INDICATORS IN THE RURAL SECTOR
}

\section{${ }^{1}$ Héctor Fabio López Castaño, 2 Jéssica Almeida Braga, ${ }^{3}$ Max Brandão de Oliveira}

\author{
${ }^{1}$ Ingeniero agrónomo, M.Sc. en Administración Financiera, Universidad EAFIT- Popayán, Cauca, (Colombia). \\ ${ }^{2}$ Estadística, M.Sc. en Gerencia Empresarial, Universidad Nacional Abierta \\ y a Distancia (UNAD), Pereira, Risaralda, (Colombia). \\ ${ }^{3}$ Estadístico, M.Sc. en Logística e Investigación Operacional (Brasil), Doctorado en Ciencias \\ Animal (Brasil), Universidade Federal do Piauí, Teresina, Piauí, (Brasil). \\ ${ }^{1}$ hflopezc@eafit.edu.co; ${ }^{2}$ jessica.almeida@unad.edu.co; \\ ${ }^{3}$ maxbrandao@ufpi.edu.br
}

\section{RESUMEN}

Contextualización: en el artículo se aborda la aplicación de técnicas de análisis de datos multivariados en el sector agro, con el propósito de caracterizar a dos grupos de agricultores de aguacate hass, a partir de los datos que recogen las entidades que tradicionalmente prestan asistencia técnica rural en el país, las unidades municipales de asistencia técnica (UMATAS) y facilitar la toma de datos en campo y arrojando luces sobre la creación de nuevos indicadores de desempeño.
Vacío de conocimiento: Hay una deficiencia en la aplicación de métodos e instrumentos de análisis de datos en el proceso de asistencia técnica rural, que permitan definir recomendaciones de actividades, insumos y manos de obra más específicas a la realidad de las distintas asociaciones o individuos de productores agrícolas

Propósito del estudio: 1) analizar los datos recogidos a través del instrumento de diagnóstico a las fincas del Registro de Usuarios de 
Asistencia Técnica (RUAT) que realizan las UMATAS en cumplimiento de su papel de acompañamiento técnico rural; 2) aplicar las técnicas estadísticas de clasificación y organización de datos más pertinente a dicho instrumento de caracterización; y 3) determinar la importancia que cumplen las variables que se recogen en los instrumentos de diagnóstico a las fincas con el propósito de construir indicadores.

Metodología: a partir de la revisión de varios referentes teóricos sobre los pasos necesarios para la creación y selección de indicadores, se siguieron las siguientes etapas metodológicas: desarrollo de un marco conceptual; selección de variables; normalización de los datos; análisis multivariado y ponderación de la información. Otros pasos posteriores, como la agregación de la información, y el análisis de robustez y sensibilidad, podrán ser parte de subsiguientes trabajos.

Resultados y conclusiones: se encontró que las variables que tuvieron mayor peso en la caracterización y agrupación de los productores fueron las pertenecientes al grupo de las buenas prácticas agrícolas y al de la producción, demostrando que con solo 8 variables se puede tener una buena aproximación a la caracterización de los productores, en lugar de las más de 36 variables de las que se compone el instrumento de diagnóstico RUAT.

Palabras clave: aguacate; análisis de datos; asistencia técnica; asociaciones de agricultores

\section{ABSTRACT}

Contextualization: This article addresses the application of multivariate data analysis techniques in the agricultural sector, in order to characterize two groups of hass avocado farmers, based on data collected by entities that traditionally provide rural technical assistance in the country, the municipal technical assistance units (UMATAS), as well as to facilitate data collection in the field and shedding light on the creation of new performance indicators.

Knowledge gap: Poor application of data analysis methods and instruments in the rural technical assistance process, which allow defining recommendations for activities, inputs and labor more specific to the reality of the different associations or individuals of agricultural producers.

Purpose: 1) Analyze the data collected through the diagnostic instrument to the Registry of Users of Technical Assistance (RUAT) farms, which is used by the UMATAS to fulfill their role of rural technical support; 2) Apply the statistical techniques of classification and organization of data most pertinent to said characterization instrument; and 3) Determine the importance of the variables included in the diagnostic instruments for farms for the construction of indicators.

Methodology: Based on the review of several theoretical references on the steps necessary for the creation and selection of indicators, the following methodological steps were followed: Development of a conceptual framework; selection of variables; normalization of data; multivariate analysis and weighting of information. Other subsequent steps such as information aggregation, robustness and sensitivity analysis, may be part of subsequent work.

Results and conclusions: It was found that the variables that had greater weight in the characterization and grouping of producers were those belonging to the group of good agricultural practices and to that of production, demonstrating that with only 8 variables it is possible to have a good approximation to the characterization of the producers, instead of the more than 36 variables that make up the diagnostic instrument RUAT.

Keywords: Avocado; data analysis; farmers associations; technical aid. 


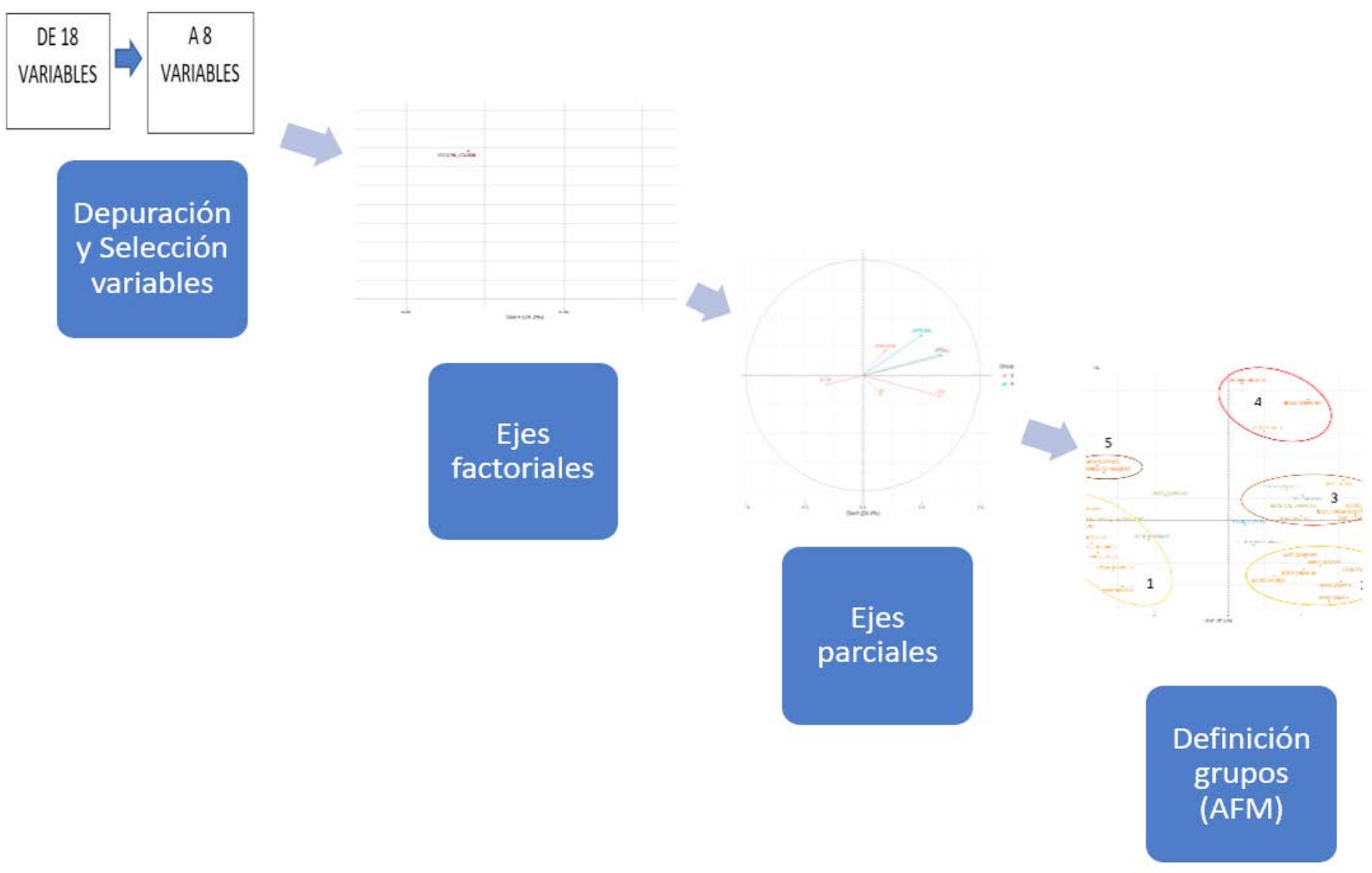

RESUMEN GRÁFICO: etapas de la elaboración del trabajo, que inicio por la selección y depuración de las variables a partir de la base de datos recolectada a través del RUAT y la plataforma Kanpo y finaliza con la definición de las características comunes de los grupos de productores, encontrados a partir del uso de la aplicación de estadística multivariada

\section{INTRODUCCIÓN}

El aguacate hass es, sin duda, un cultivo muy atractivo para Popayán y el departamento del Cauca en general, donde según datos de la Corporación Colombia Internacional (Idrobo, 2013), existe potencial para exportar a Italia, España, Francia, China, Korea, Abu Dabi y Estados Unidos, favoreciendo a más de 520 familias en el Cauca, representadas en más de 1.000 hectáreas sembradas en el departamento y 420 hectáreas sembradas en Popayán. Es por esto que buscar estrategias que favorezcan la toma de decisiones con respecto a aguacate hass para pequeños productores es una tarea que en definitiva favorece de manera sostenible al territorio.
Experiencias exitosas como Kanpo y CAMPO UNAD, las cuales "Proponen el desarrollo de múltiples servicios de consultoría, en temas relacionados con la productividad agropecuaria, las asesorías ambientales" (Leal, 2016, p.67), constituyen una importante opción para captar datos de la actividad agrícola y ser procesada para generar indicadores de desempeño (key performance index, KPI por sus siglas en inglés). La importancia de los indicadores radica en que, de acuerdo con Solá \& María (2003), un indicador permite seguir y evaluar en el tiempo, que son los aspectos más importantes para una organización, mediante su comparación con referentes externos e internos. 
La anterior definición implica que un indicador debe cumplir dos funciones principales: la primera, describir la situación actual de un proyecto, acción, programa o cualquier fenómeno; y la segunda, ayudar a emitir un juicio de valor sobre el desempeño actual de dicho fenómeno.

Por ejemplo, si se tiene en cuenta el rendimiento por hectárea como un indicador de productividad y se supone que en una finca productora de aguacate en el municipio de El Tambo, Cauca, tiene un valor de 5 ton/ha, dicha cifra, por sí sola, no diría nada sobre su desempeño productivo, mientras que se contrasta dicho valor con el indicador de productividad de aguacate promedio para el municipio de 3.5 ton/ha, queda claro que, en efecto, la finca posee un óptimo desempeño.

Igualmente, dependiendo del tipo de fenómeno, los indicadores pueden usarse en la medición de insumos (outputs), procesos o actividades, productos (outputs), resultados finales (outcomes) o el desempeño de en las dimensiones de eficiencia, eficacia, calidad y economía (Armijo, 2011).

En Colombia se ha concebido la extensión rural como un proceso de transferencia tecnológica que implica la adopción de nuevas tecnologías por parte del mayor número de agricultores, a través de capacitaciones. En dichas capacitaciones el técnico, quien conoce el avance tecnológico, trata de persuadir al agricultor para que adopte el cambio y así obtenga mejoras en sus procesos y recompensas económicas (Clavijo, 2008).

En este sentido, Rodríguez-Espinoza (2015) propone pasar del enfoque tradicional de transferencia de tecnología a uno nuevo, basado en el desarrollo de capacidades de autogestión de abajo hacia arriba. En este nuevo enfoque, la interconexión entre agricultores y la generación de redes que facilitan el intercambio de experiencias, métodos o actividades entre los distintos actores del sistema, constituye la clave para fortalecer el desarrollo rural.

Por otra parte, la mayoría de los cultivos tropicales poseen escasa información disponible sobre dónde sembrar y cómo manejar los cultivos efectivamente (Jiménez, et al., 2009), lo cual implica un enorme número de posibles combinaciones de prácticas de manejo, así como una gran variación de las condiciones de crecimiento en las diferentes etapas de desarrollo de los cultivos.

Bajo estas condiciones de alta variación en las prácticas de manejo y en las condiciones del cultivo, cada unidad productiva se considera un evento único o experimento (Cock, 2007), lo cual conlleva a la idea de que, si fuera posible recolectar información de un gran número de eventos de cosecha bajo condiciones variadas, podría usarse esta información para desarrollar modelos que sirvan de indicadores para mejorar el manejo de los cultivos a los pequeños productores, de acuerdo a las condiciones específicas de sus fincas (Jiménez et al., 2011).

Es así como Satizábal et al. (2012), proponen modelos para caracterizar los cultivos de mora y lulo en Colombia y usar esta información para mejorar la toma de decisiones entre los pequeños productores del país, aplicando métodos de análisis de datos computacionales conocidos también como "Mapas autoorganizados" (SOM) o mapas Kohonen para la mora y el uso de redes neuronales para el lulo.

Partiendo de la hipótesis de que es posible mejorar la toma de decisiones para dos de los principales actores del sistema agroindustrial colombiano, como son las unidades 
municipales de asistencia técnica (UMATAS) y las asociaciones de productores agrícolas, a partir del análisis de datos avanzado, el presente estudio plantea tres objetivos: 1) analizar los datos recogidos a través del instrumento de diagnóstico a las fincas RUAT que realizan las UMATAS en cumplimiento de su papel de acompañamiento técnico rural; 2) aplicar las técnicas estadísticas de clasificación y organización de datos más pertinente a dicho instrumento de caracterización; y 3) determinar la importancia que cumplen para la construcción de indicadores las variables que se recogen en los instrumentos de diagnóstico a las fincas.

\section{MATERIALES Y MÉTODOS}

La metodología para la selección de indicadores propuesta por Nardo et al. (2005) se usó como referente teórico para el desarrollo del presente trabajo. Esta comprende los siguientes pasos: desarrollo de un marco conceptual, selección de variables, imputación de datos perdidos, normalización de los datos, análisis multivariado, ponderación de la información, agregación de la información, y análisis de robustez y sensibilidad.

Para identificar de forma efectiva las variables que son realmente significativas para la formación de los indicadores, se aplicó el análisis de componentes principales (ACP) como herramienta de estadística multivariante, la cual realiza una selección de los datos principales, excluyendo las variables no correlacionadas. Este tipo de análisis nos permite adelantar el proceso de selección propuesto por Nardo et al. (2005), debido a que, en el conjunto de datos utilizado en este estudio, no hubo la necesidad de imputación de datos y en la ACP se tratan los datos como variables tipificadas o expresadas en desviaciones con respecto a la media, y por esto, están correlacionadas, teniendo como principio base que no es necesario establecer jerarquías ni comprobar la normalidad para los datos cuantitativos.
Para el desarrollo del marco conceptual, se tuvieron en cuenta la relevancia, la frecuencia con que se recogen y la disponibilidad al público de las variables por recoger (Soto \& Schuschny; 2009), definiéndose que el instrumento de caracterización más adecuado para el estudio es el Registro de Usuarios de Asistencia Técnica (RUAT).

Se recopilaron los datos y las variables para trabajar en el estudio a partir del diligenciamiento del RUAT, realizado a 33 productores del municipio de Popayán por parte de la UMATA municipal, en el segundo semestre del año 2017 y a 68 productores del municipio de El Tambo, entrevistados por dos estudiantes del programa de ingeniería agronómica de la UNAD, CEAD Popayán, en el primer semestre del año 2018.

Se compararon ambas bases de datos para hacerlas coincidir, obteniéndose una base de datos de 18 variables. Posteriormente, con el propósito de obtener la carga factorial más significativa para los datos, se siguió lo recomendado por De la Fuente (2011) y se fueron eliminando progresivamente columnas de la base de datos consolidada, principalmente del grupo sociodemográfico (DEMOG), hasta alcanzar la carga factorial global más significativa para el estudio, es decir, el $43.4 \%$ correspondiente a 8 variables para la base de datos de El Tambo y el $51.6 \%$ para la base de datos de Popayán.

De esta manera las bases de datos se dividieron en 4 grupos principales de variables: 4 económicas (ECON_CUAN), 2 productivas (PT), DEMOG y 1 de certificación en BPA (BPA), tal como se muestra en la tabla 1.

Aunque autores como Gonzales-García \& Taborda (2015), destacan el uso del análisis de componentes principales (ACP) para encontrar 
relaciones ocultas entre las variables que movilizan el sistema (principales) con otras que permanecen ocultas al simple análisis visual (latentes). En este trabajo se aplicó la técnica del análisis factorial múltiple (AFM), propuesta por Escofier \& Pagès (2008) y Pages (2004), para analizar bases de datos en las que un conjunto de individuos (Xi), está descrito por varios conjuntos de variables $(\mathrm{Ki})$, que pueden ser del tipo cuantitativo o cualitativo. Los datos se ejecutaron a través del software $R$ Studio versión 3.5.3, donde fue necesario instalar las librerías FactoMineR, Factoextra, Shiny y Factoshiny.

Tabla 1. Variables y grupos de variables involucradas en el estudio

\begin{tabular}{|c|c|c|c|}
\hline Grupo & Variables & Tipo & Sigla \\
\hline \multirow{4}{*}{ Económicas } & Ingreso familiar total anual & Cuantitativa & IFTA \\
\hline & $\begin{array}{l}\text { Ingreso familiar total anual derivado } \\
\text { de la actividad agrícola }\end{array}$ & Cuantitativa & IFTAA \\
\hline & Costos establecimiento por hectarea & Cuantitativa & CE \\
\hline & Precio de venta promedio por kg & Cuantitativa & PV \\
\hline \multirow{2}{*}{ Productivas } & Área en aguacate & Cuantitativa & AREAA \\
\hline & Producción total & Cuantitativa & PT \\
\hline BPA & Certificación en BPA & Cualitativa & BPA \\
\hline Sociodemográficas & Sitio de venta & Cual. & SV \\
\hline
\end{tabular}

Fuente: Autores

Según la estructura visualizada en los análisis factoriales múltiples individuales (Figuras 1 y 2), se identificó la necesidad de trabajar las variables por grupos. Estas variables se separaron en grupos de variables demográficas, económicas, productivas y de certificación. Las variables de certificación son las que identifican la existencia de una certificación de buenas prácticas agrícolas (BPA) y certificación de registro de un predio exportador.

Posteriormente, se realizó el análisis factorial múltiple para los individuos a través del gráfico de individuos, obteniéndose grupos de productores que presentan comportamientos correlacionados entre ellos.
Por último, se empleó el mapa de los ejes parciales, con el fin de visualizar cómo los factores generados por los análisis separados del grupo de variables están relacionados con los factores del análisis factorial múltiple, que es la representación gráfica del análisis de componentes principales.

\section{RESULTADOS Y DISCUSIÓN}

\subsection{Conformación de los ejes factoriales}

Para el caso de El Tambo, se encontró que el grupo de variables ECON_CUAN y PDCN ofrecen la mayor carga de inercia sobre el eje 1 , con un $24.4 \%$, mientras que el grupo de variables DEMOG y BPA aportaron la mayor carga de inercia para formar el eje 2 , con un $19 \%$, tal como lo muestra la Figura 1. 


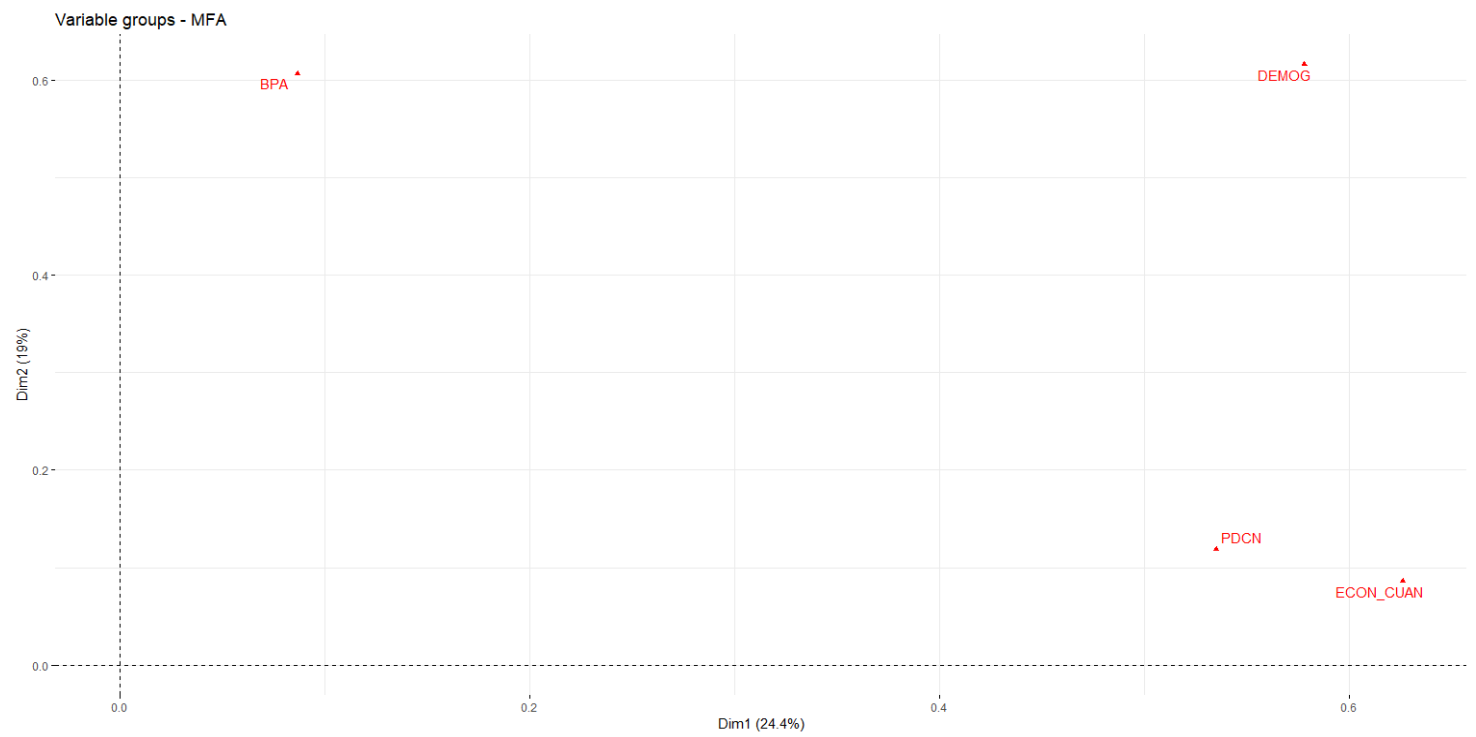

Figura 1. Contribuciones de los grupos de variables a la formación de los ejes, Municipio de El Tambo.

Fuente: Autores

Para el caso de Popayán (Figura 2), aunque el de variables PDCN (31.2\%) y de BPA para el eje grupo de variables DEMOG tuvo el mayor apor- 2 (20.4\%), con el propósito de evitar confusiote a la carga de inercia global, se prefirió definir nes en la interpretación de la agrupación de los la carga de inercia sobre el eje 1 con el grupo individuos hecha a través del AFM.

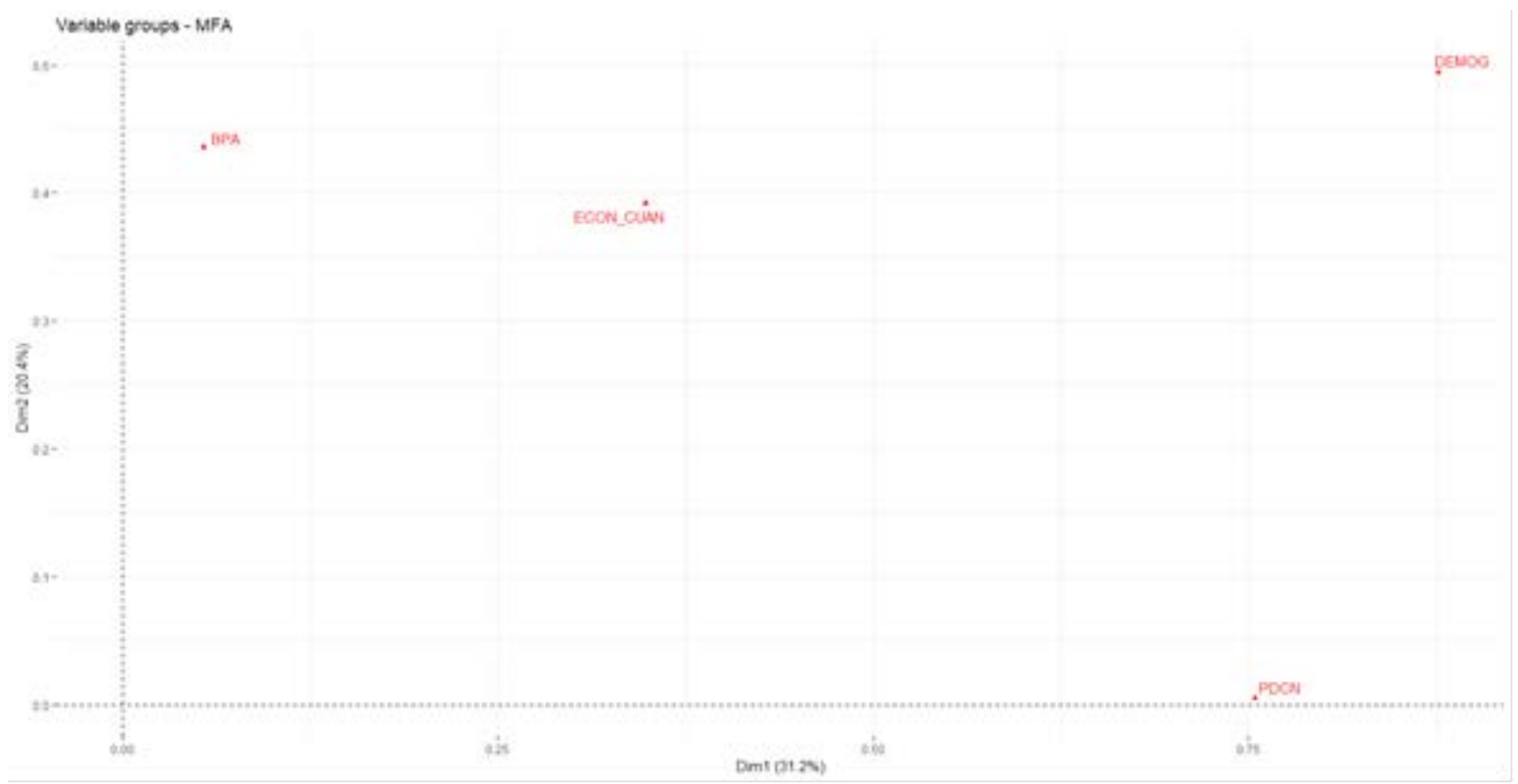

Figura 2. Contribuciones de los grupos de variables a la formación de los ejes para el municipio de El Tambo. 


\subsection{Conformación ejes parciales}

El mapa de los ejes parciales para El Tambo (Figura 3), muestra una correlación altamente positiva entre las variables PT e IFTAA y positiva entre estas y AREAA, y una correlación negativa con relación a IFTA. Del mismo modo, se observó que las distancias entre las variables de Área utilizadas en Aguacate en hectáreas (AREAA), Producción total medida en kilogramo por hectárea/año (PT), Ingreso familiar total anual derivado exclusivamente de la actividad agropecuaria (IFTAA), costo sostenimiento por hectárea (CS), Precio de venta por productores (preciovp) y su origen, muestran una buena calidad de representación del estudio.

Para el municipio de Popayán, la Figura 4, muestra que en el eje 2 las variables IFTAA e IFTA, están fuertemente correlacionadas entre ellas, así como las variables PT, AREAA y PRECIOVP en el eje 1. Por último, al igual que para el caso de Popayán, la distancia entre estas variables y su origen muestran también una buena calidad de representación del estudio.

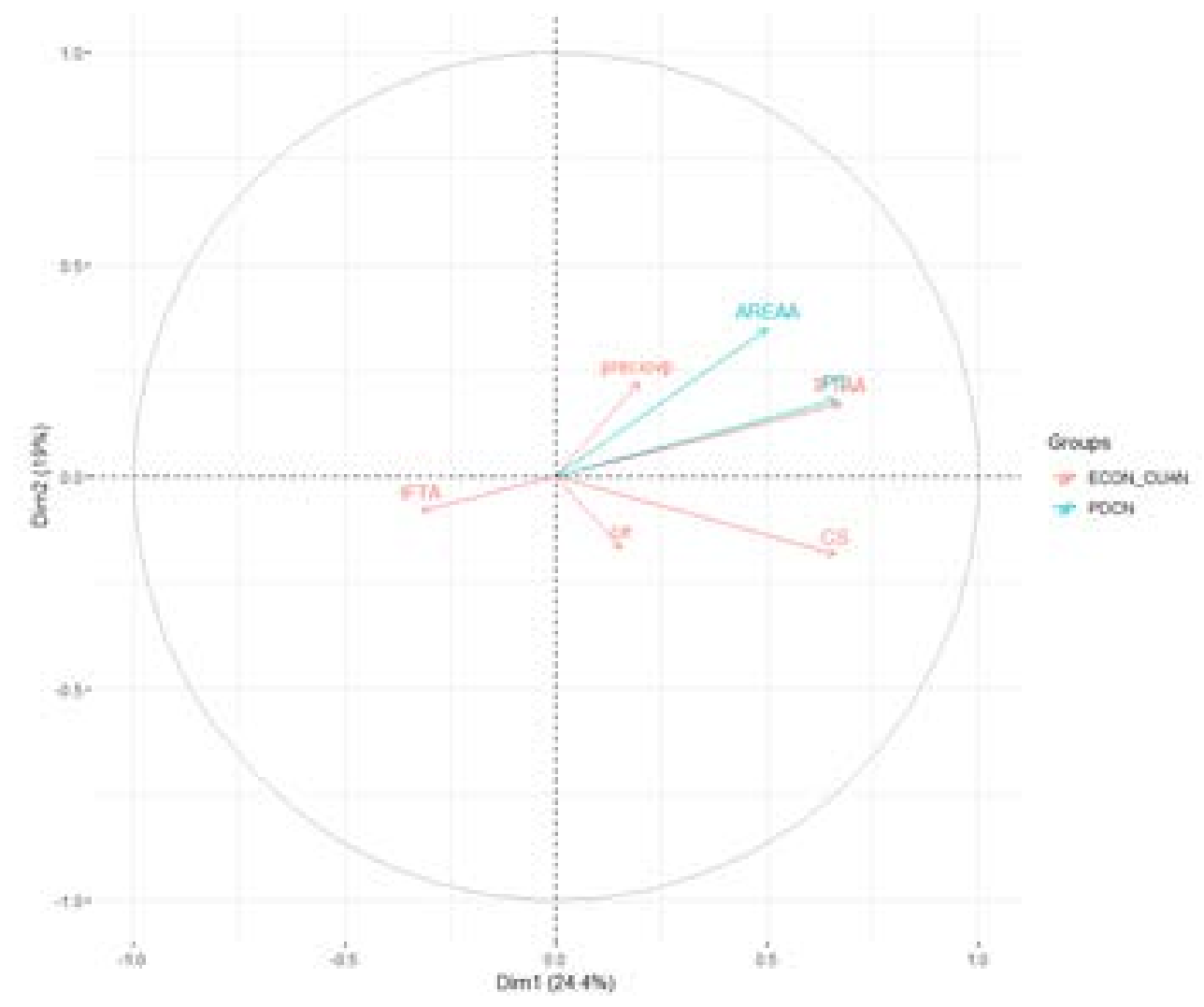

Figura 3. Gráfico de Análisis Factorial Múltiple de las variables del municipio El Tambo.

Fuente: Autores 


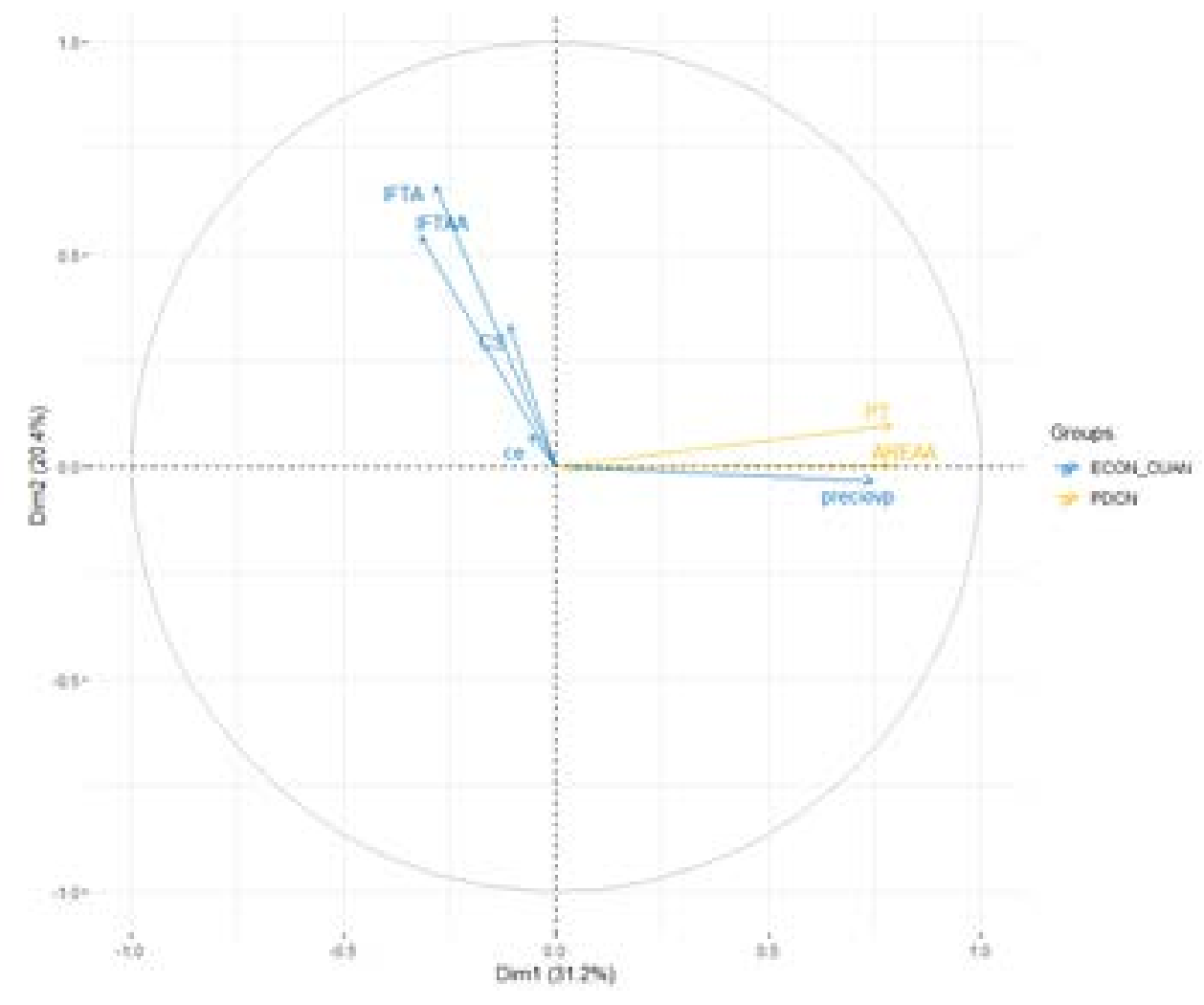

Figura 4. Gráfico de Análisis Factorial Múltiple de las variables del municipio El Tambo.

Fuente: Autores

\subsection{Análisis factorial múltiple}

Para el municipio El Tambo (Figura 5), las fincas del grupo (1), son las que mostraron valores más altos para las variables PV, CS, CE, SV y BPA, lo cual demuestra que una mayor productividad y un mayor precio de venta pueden estar relacionados con ventas a clientes institucionales gracias a las certificaciones en BPA y a una mayor inversión en el cultivo.

El grupo (2), se destaca por tener la más baja AREA (1 ha) aunque el segundo mayor rendimiento $(3.035 \mathrm{Kg} / \mathrm{ha})$, al igual que el segundo valor más alto de IFTA (COP \$7.629.412), mientras que el grupo (3) de productores se destaca por tener la segunda mayor área sembrada en aguacate (AREAA), aunque el más bajo rendimiento de todos (COP $\$ 2.508$ ton/ha), lo que coincide con las menores CS, CE y IFTAA de COP $\$ 1.063 .712 /$ ha, $\$ 2.539 .474 /$ ha y COP $\$ 1.294 .737 / \mathrm{ha}$, respectivamente.

El grupo (4) se destaca por tener la mayor AREAA promedio (2) aunque con un rendimiento bajo de 2.508 ton/ha y ninguna certificación en BPA, aunque sí presentan el PRECIOVP más alto, posiblemente debido a una situación coyuntural donde su cosecha coincide con los picos más altos de venta; por último, mientras que el grupo (5) de productores se destaca por tener las siembras más recientes, lo cual se refleja en que no presenta aún PT, PRECIOVP y IFTAA; el hecho que el $66.6 \%$ de ellos tenga un nivel educativo superior, muestra que son productores que no dependen exclusivamente de la actividad agrícola para sobrevivir. 


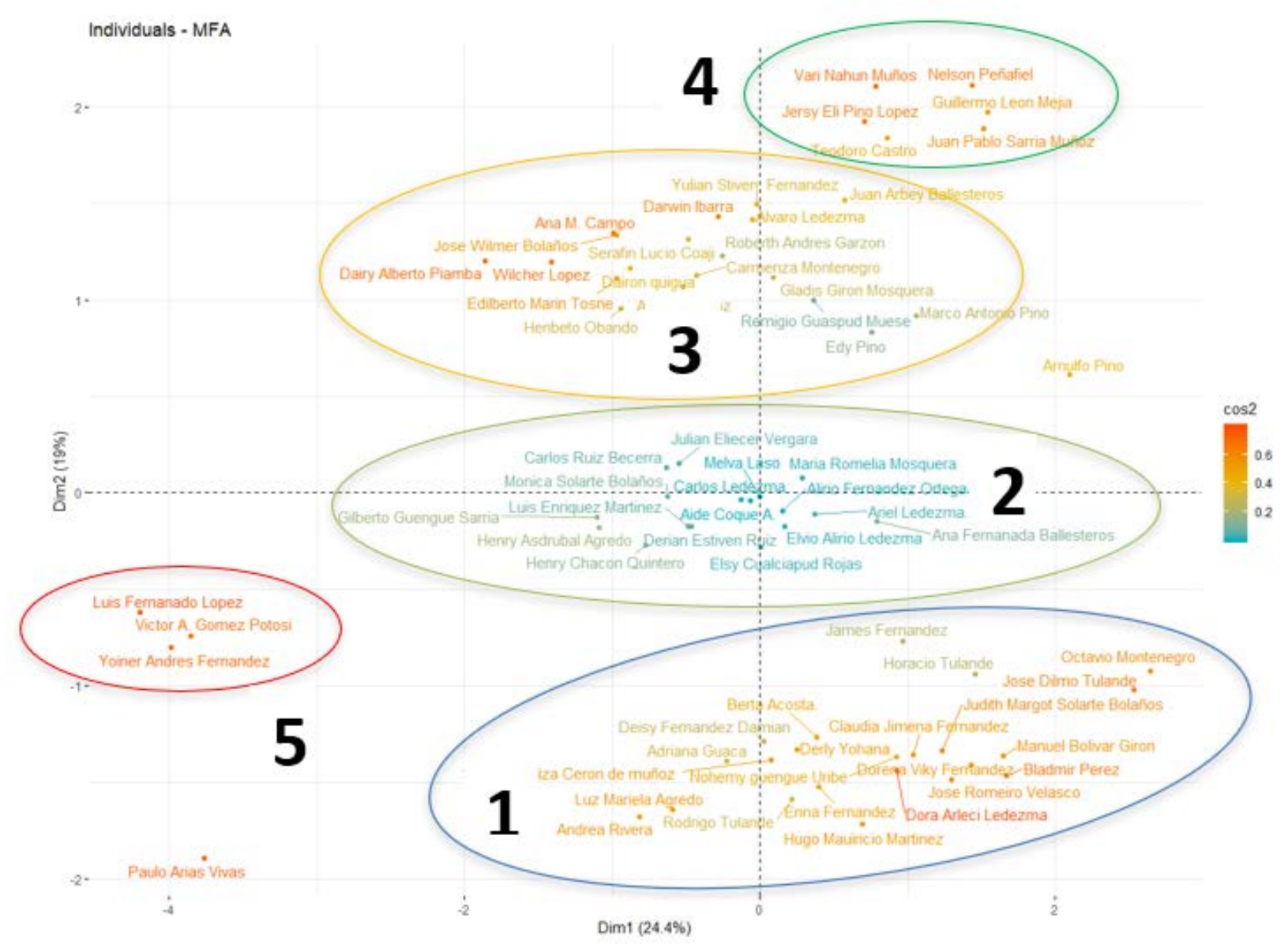

Figura 5. Gráfico de Análisis Factorial Múltiple por individuos, productores en el estudio del municipio El Tambo.

Fuente: Autores

Para el caso de Popayán, (Figura 6), se observó una agrupación entre los productores más influenciada por las variables productivas y BPA donde se nota la influencia de los grupos de variables que conforman los ejes factoriales; de este modo, el grupo (1) no posee certificaciones en BPA ni buenas características productivas, mientras que el grupo (2) se destaca por tener buenas características productivas y vender sus productos al mercado informal, pero no están certificados en BPA, mientras que el grupo (5) sí está certificado en BPA, pero con bajas características productivas y ventas nulas pues tienen siembras recientes. Por otra parte, el grupo (3) comparte con el grupo (2) unas buenas características productivas al igual que la mayoría de las ventas en el mercado informal, pero la mayoría si están certificados en BPA. Por último, el grupo (4), es el que presenta mayores valores productivos, económicos y de BPA, coincidiendo con la orientación de los ejes factoriales. 


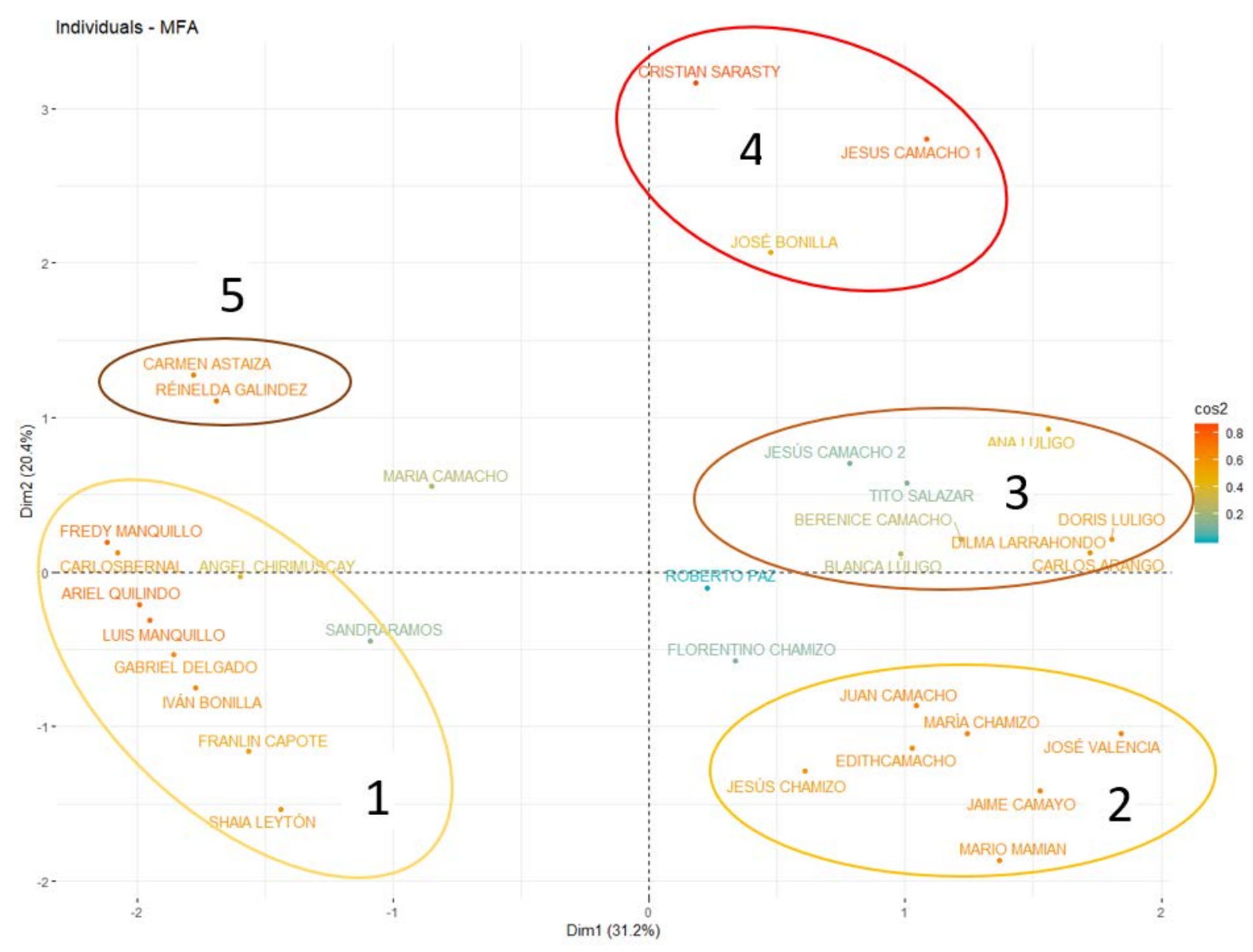

Figura 6. Gráfico de Análisis Factorial Múltiple por individuos, productores en el estudio del municipio de Popayán.

Fuente: Autores

\section{CONCLUSIONES}

Aunque las entidades de apoyo a la agricultura cuentan con instrumentos de diagnóstico de las fincas como el RUAT y las listas de chequeo en BPA del ICA, que contienen un amplio número de variables en distintas áreas transversales, se requiere mejorar el manejo de estos datos para generar información de valor para el sector.

Con los resultados obtenidos a través de los análisis estadísticos múltiples aplicados, se encontró que, de las dieciocho variables colectadas de los productores, ocho de ellas pueden ser utilizadas para la formación de otros tipos de indicadores de ayuda en la búsqueda de estrategias que favorezcan la toma de decisiones con respecto a aguacate hass para pequeños productores de manera sostenible al territorio.

Al mismo tiempo que se analizan indicadores de desempeño más exactos para el sector agrícola, se hace necesaria la implementación de sistemas de información que faciliten la captura, almacenamiento y procesamiento de los datos obtenidos de las fincas y su interacción con los asistentes técnicos. 
Se espera que a medida que se acumule una mayor cantidad de datos de las fincas, la robustez de las pruebas y de los modelos como el de Tukey y la Regresión Lineal mejoren, permitiendo establecer indicadores de mayor confiabilidad.

El uso de técnicas de análisis multivariante como clúster, análisis de componentes principales y Biplot, se perfilan como las mejores herramientas para representar las interrelaciones entre las variables de interés y las observaciones.

Se resalta también la necesidad de sensibilizar a los productores y a las entidades de acompañamiento a la agricultura en la importancia de recoger y usar los datos de sus actividades como insumo principal para mejorar el desempeño de las fincas y la toma de decisiones. Así, por ejemplo, en un diagnóstico realizado a 13 UMATAS del departamento del Cauca en el año 2016, se evidenció que los factores más críticos para estas entidades son el recurso humano, la información y tecnología para la asistencia técnica y la planeación del desarrollo rural (Salamanca, 2014).

\section{CONTRIBUCIÓN DE LA AUTORÍA}

Primer autor: Metodología, investigación, análisis de datos, escritura del borrador original. Segundo autor: Conceptualización, análisis de datos, revisión y edición. Tercer autor: Revisión y edición.

\section{AGRADECIMIENTOS}

Agradecimientos al programa Innovación Cauca a través del cual se financió el proyecto de investigación: "Optimización del proceso de asistencia técnica y aumento de la productividad para agricultores de zonas rurales de Popayán y el Tambo, Cauca, a través de la articulación de la plataforma tecnológica web y móvil Kanpo y UNAD" el cual dio origen al actual trabajo, a la UNAD por el personal de apoyo, a la empresa Kanpo SAS por poner a su disposición su plataforma web y móvil para la captura de datos, a Oscar Castaño por el apoyo en el procesamiento de las bases de datos e interpretación del AFM, a Jesús Camacho y Manuel Bolívar Girón por abrirnos las puertas de sus asociaciones, a Ana Julieth Caldón, Ana Fernanda Ballesteros y Juan Pablo Fernández por el apoyo en el trabajo de campo.

\section{LITERATURA CITADA}

Armijo, M. (2011). Planificación estratégica e indicadores de desempeño en el sector público. CEPAL, Serie Manuales, No. 69. Recuperado de http://bibliotecadigital.seplan.planejamento.gov.br/bitstream/handle/iditem/504/06\%20 Planificacion\%20y\%20indicadores_ILPES. pdf?sequence $=1$

Cock, J. (2007). Sharing commercial information. In: Innovation Workshop for the Agricultural Sector: Site Specific Agriculture based on Sharing Farmers Experiences, CIAT, Cali, Colombia, October.

Clavijo, N. (2008). Transferencia de tecnología [en línea]. Colección apuntes de clase. Bogotá, D.C.: Editorial Pontificia Universidad Javeriana. Recuperado de https://isfcolombia.uniandes.edu.co/ images/documentos/9dejulioclavijo.pdf

De la Fuente, S. (2011). Análisis factorial. Facultad de Ciencias Económicas y Empresariales. Universidad Autónoma de Madrid. Recuperado de http://www.fuenterrebollo.com/Economicas/ ECONOMETRIA/MULTIVARIANTE/FACTORIAL/ analisis-factorial.pdf

Escofier, B., y Pagès, J. (2008). Analyses factorielles simples et multiples. Objectifs méthodes et interprétation (pp. 328-p). Dunod.

Gonzales-García, N., \& Taborda, A. (2015). Análisis de componentes principales SPARSE. Formulación, algoritmos e implicaciones en el análisis de datos. [Tesis de maestría]. Salamanca, España: Universidad de Salamanca.

Idrobo, C. (2013). Aguacate Hass, un motor de desarrollo en el Cauca. Diario del Cauca. Recuperado de http://diariodelcauca.com.co/aguacate-hassun-motor-de-desarrollo-en-el-cauca-90481

Jiménez, D., Cock, J., Satizábal, H. F., Pérez-Uribe, A., Barreto, M.A., Pérez-Uribe, A., ....... Van Damme, P. (2009). Analysis of andean blackberry 
(rubus glaucus) production models obtained by means of artificial neural networks exploiting information collected by small-scale growers in colombia and publicly available meteorological data. Computers and electronics in agriculture, 69(2), 198-208. https://doi.org/10.1016/j.compag.2009.08.008

Jiménez, D., Cock, J., Jarvis, A., Garcia, J., Satizábal, H. F., Van Damme, P., y Barreto-Sanz, M. A. (2011). Interpretation of commercial production information: A case study of lulo (Solanum quitoense), an under-researched Andean fruit. Agricultural systems, 104(3), 258-270. https://doi. org/10.1016/j.agsy.2010.10.004

Leal, J. (2016). CAMPO UNAD. Sembrando un país en red para la paz [en línea]. Recuperado de https://www.unad.edu.co/

Nardo, M., Saisana, M., Saltelli, A., Tarantola, S., Hoffmann A., Giovannini E. (2005), "Handbook on Constructing Composite Indicators: Methodology and User Guide", OECD Statistics Working Papers, 2005/03, OECD Publishing, Paris. http:// dx.doi.org/10.1787/533411815016

Rodríguez-Espinoza, H., Ramírez-Gómez, C., y Restrepo-Betancur, L. (2015). Nuevas tendencias de la extensión rural para el desarrollo de capacidades de autogestión. Corpoica Ciencia y Tecnología Agropecuaria, 17(1), 31-42.

Salamanca, M. (2014). Diagnóstico de 17 UMATAS del departamento del Cauca. Convenio ESAPMADR 2014. Colombia: Escuela Superior de Administración Pública, ESAP.

Satizábal, H., Barreto-Sanz, M., Jiménez, D., PérezUribe, A., y Cock, J. (2012). Enhancing decisionmaking processes of small farmers in tropical crops by means of machine learning models. In Technologies and Innovations for Development, pp. 265-277. Springer.

Solà, J. M. G., y María, J. (2003). Indicadores de gestión para las entidades públicas. en VIII Congresso Internacional del Clad sobre la reforma del estado y de la administración pública, Panamá (pp. 28-31).

Soto, H., y Schuschny, A. R. (2009). Guía metodológica: diseño de indicadores compuestos de desarrollo sostenible. Recuperado de https://repositorio.cepal.org/bitstream/handle/11362/3661/ $\underline{\text { S2009230_es.pdf?sequence }=1 \& \text { isAllowed }=y}$

Pages, J. (2004). Multiple factor analysis: Main features and application to sensory data. Revista Colombiana de Estadística, 27(1), 1. 
